\title{
Dynamics and Stability of Permanent-Magnet Synchronous Motor
}

\author{
Ren He and Qingzhen Han \\ School of Automotive and Traffic Engineering, Jiangsu University, Zhenjiang 212013, China \\ Correspondence should be addressed to Ren He; heren1962@163.com
}

Received 20 February 2017; Accepted 7 May 2017; Published 15 June 2017

Academic Editor: Kishin Sadarangani

Copyright ( 2017 Ren He and Qingzhen Han. This is an open access article distributed under the Creative Commons Attribution License, which permits unrestricted use, distribution, and reproduction in any medium, provided the original work is properly cited.

\begin{abstract}
The aim of this article is to explore the dynamic characteristics and stability of the permanent-magnet synchronous motor (PMSM). PMSM equilibrium local stability condition and Hopf bifurcation condition, pitchfork bifurcation condition, and fold bifurcation condition have been derived by using the Routh-Hurwitz criterion and the bifurcation theory, respectively. Bifurcation curves of the equilibrium with single and double parameters are obtained by continuation method. Numerical simulations not only confirm the theoretical analysis results but also show one kind of codimension-two-bifurcation points of the equilibrium. PMSM, with or without external load, can exhibit rich dynamic behaviors in different parameters regions. It is shown that if unstable equilibrium appears in the parameters regions, the PMSM may not be able to work stably. To ensure the PMSMs work stably, the inherent parameters should be designed in the region which has only one stable equilibrium.
\end{abstract}

\section{Introduction}

Permanent-magnet synchronous motor (PMSM) is one of the most important development trends in a variety of driving motors, due to its low inertia, low noise, high power density, and high efficiency. It has both the advantages of reliable operation of AC motor and the advantages of excellent speed control performance of DC motor which is very suitable for engineering application [1-4]. PMSM is a multivariable, strongly nonlinear, and strongly coupled electromechanical system $[5,6]$. When PMSM is used in engineering, the parameters are not fixed but fluctuate within a range according to the changes of working environment, and the torque and speed may irregularly oscillate in some parameter regions which is not allowed for ensuring the stability and security. So the dynamic behaviors of the PMSM within different parameter regions as well as the condition of stability should be generally discussed to provide theoretical basis for the use of PMSM.

Current domestic and foreign scholars have done a lot of research on the dynamic characteristics and stability of PMSM. For example, small-signal equations of the MCPMSM system, including both power stage and control stage, are derived in [7] and stable region and its boundary are obtained according to a Lyapunov-type stability analysis based on these equations. Li et al. [8] deduced the smallsignal model of the open-loop control system of threephase PMSM, and the effect of speed, stator resistance, stator inductance, excitation magnetic linkage, moment of inertia, and load on the system stability is studied in detail by using stability criterion of eigenvalues of the state transition matrix in the linear system theory. Hinkkanen et al. [9] proposed a reduced-order position observer with stator-resistance adaptation for motion sensorless permanent-magnet synchronous motor drives and the effect of inaccurate model parameters on the local stability of the position estimation is studied by analyzing the small-signal stability of the nonlinear estimation-error dynamics model. The stability of sensorless control of PMSMs (assume the position and the electromagnetic torque are known and the load is constant) are studied by making a strict Lyapunov function for the error system [10]. The stability of the PMSM-controller system with time delay, as the mechanical time constant is significantly higher than the electrical time constant in the PMSM-controller system, is studied by root locus in the complex plane [11]. 
With the large variation in inertia due to varying load and parametric uncertainty, Yadav et al. [12] discussed the relative robust stability of PMSM with parameter uncertainty by using Kharitonov theorem and Routh stability criterion. The transfer function of PMSM drives system with feedforward complete decoupling is developed based on the small-signal model in [13], and the stable operation range of system and PI parameter of flux weakening control are analyzed by Routh stability criterion. In $[14,15]$, the unstable dynamic behaviors of PMSM, used in EV, without external inputs or load are observed over the entire range of singleparameter values in the bifurcation diagram. The chaotic motions (unstable) of the PMSM during the EV startingrunning process with and without large amplitude oscillation load are discussed in [16], respectively. Analysis of the above literature shows that current domestic and foreign scholars have done a lot of research on the dynamic characteristics of PMSM, but insufficiency still exists, such as limited work on the variation regularity of the dynamics and stability with multiple parameter variations and the relationship between local and global dynamics of PMSM.

This paper aimed at exploring the dynamic characteristics and stability of PMSM by nonlinear dynamics method. The stability conditions and the bifurcation conditions of the equilibrium points have been derived. The bifurcation curves of the equilibrium with single and double parameters are obtained by continuation method. The local dynamic characteristics of the equilibrium points and the global dynamic characteristics of PMSM are discussed by bifurcation set and bifurcation diagram, respectively.

\section{Dynamic Model of PMSM}

The dynamic model of PMSM can be described as the following three-dimensional nonlinear system $[17,18]$ :

$$
\begin{aligned}
& \frac{\mathrm{d} i_{d}}{\mathrm{~d} t}=\frac{\left(u_{d}-R_{1} i_{d}+\omega L_{q} i_{q}\right)}{L_{d}} \\
& \frac{\mathrm{d} i_{q}}{\mathrm{~d} t}=\frac{\left(u_{q}-R_{1} i_{q}-\omega L_{d} i_{d}+\omega \psi_{f}\right)}{L_{q}} \\
& \frac{\mathrm{d} \omega}{\mathrm{d} t}=\frac{\left[n_{p} \psi_{f} i_{q}+n_{p}\left(L_{d}-L_{q}\right) i_{d} i_{q}-T_{L}-\beta \omega\right]}{J},
\end{aligned}
$$

where $i_{d}, i_{q}$, and $\omega$ represent the $d$-q axis stator current and motor angular speed, respectively; $u_{d}$ and $u_{q}$ are the $d$ - $q$ axis stator voltage components, respectively, $L_{d}$ and $L_{q}$ are the $d-q$ axis stator inductors, respectively, and $R_{1}, \psi_{f}, \beta, J, n_{p}$, and $T_{L}$ are the stator winding resistance, the permanent-magnet flux, the viscous damping coefficient, the polar moment of inertia, the number of pole-pairs, and the external load torque, respectively.

By applying an affine transformation and a time-scaling transformation, system (1) can be transformed into the following dimensionless model:

$$
\begin{aligned}
& \frac{\mathrm{d} \tilde{i}_{d}}{\mathrm{~d} \widetilde{t}}=-\frac{L_{q} \widetilde{i}_{d}+\widetilde{\omega} \tilde{i}_{q}+\widetilde{u}_{d},}{L_{d}} \frac{\widetilde{i}_{q}}{\mathrm{~d} \tilde{t}}=-\widetilde{i}_{q}-\widetilde{\omega}_{d}+\gamma \widetilde{\omega}+\widetilde{u}_{q}, \\
& \frac{\mathrm{d} \widetilde{\omega}}{\mathrm{d} \widetilde{t}}=\sigma\left(\widetilde{i}_{q}-\widetilde{\omega}\right)+\varepsilon \widetilde{i}_{d} \widetilde{i}_{q}-\widetilde{T}_{L},
\end{aligned}
$$

where $\gamma=n_{p} \psi_{f}^{2} / R_{1} \beta ; \sigma=L_{q} \beta / R_{1} J ; \tilde{u}_{q}=n_{p} L_{q} \psi_{f} u_{q} / R_{1}^{2} \beta$; $\tilde{u}_{d}=n_{p} L_{q} \psi_{f} u_{d} / R_{1}^{2} \beta ; \varepsilon=L_{q} \beta^{2}\left(L_{d}-L_{q}\right) / L_{d} J n_{p} \psi_{f}^{2} ; \widetilde{T}_{L}=$ $L_{q}^{2} T_{L} / R_{1}^{2} J$.

\section{Equilibrium Point and Bifurcations}

(A) In order to study the inherent characteristics of the smooth-air-gap PMSM, namely, $L_{d}=L_{q}, \widetilde{u}_{d}=0, \widetilde{u}_{q}=0$, and $\widetilde{T}_{L}=0$, system (2) is written as

$$
\begin{aligned}
& \frac{\mathrm{d} \widetilde{i}_{d}}{\mathrm{~d} \widetilde{t}}=-\tilde{i}_{d}+\widetilde{\omega}_{q}, \\
& \frac{\mathrm{d} \tilde{i}_{q}}{\mathrm{~d} \tilde{t}}=-\widetilde{i}_{q}-\widetilde{\omega}_{d}+\gamma \widetilde{\omega}, \\
& \frac{\mathrm{d} \widetilde{\omega}}{\mathrm{d} \widetilde{t}}=\sigma\left(\widetilde{i}_{q}-\widetilde{\omega}\right) .
\end{aligned}
$$

There are only two combined parameters, $\gamma$ and $\sigma$, in (3), and each parameter represents multiple parameters of the actual PMSM. These two parameters are assumed to be totally independent of each other for further discussing the dynamic characteristics in the following text. The actual dynamic behaviors can be obtained by substituting the actual parameters into $\gamma$ and $\sigma$.

By the analysis of the equilibrium points, $\left(\widetilde{i}_{d_{0}}, \widetilde{i}_{q_{0}}, \widetilde{\omega}_{0}\right)$, of system (3), the equilibrium points equations are obtained:

$$
\left\{\begin{array} { l } 
{ - \widetilde { i } _ { d _ { 0 } } + \widetilde { \omega } _ { 0 } \widetilde { i } _ { q _ { 0 } } = 0 } \\
{ - \widetilde { i } _ { q _ { 0 } } - \widetilde { \omega } _ { 0 } \widetilde { i } _ { d _ { 0 } } + \gamma \widetilde { \omega } _ { 0 } = 0 } \\
{ \sigma ( \widetilde { i } _ { q _ { 0 } } - \widetilde { \omega } _ { 0 } ) = 0 }
\end{array} \Longrightarrow \left\{\begin{array}{l}
\widetilde{i}_{d_{0}}=\widetilde{\omega}_{0}^{2} \\
\widetilde{i}_{q_{0}}=\widetilde{\omega}_{0} \\
\widetilde{\omega}_{0}\left(\widetilde{\omega}_{0}^{2}-\gamma+1\right)=0 .
\end{array}\right.\right.
$$

Solving the equilibrium points equations, we obtain the following:

(1) If $\gamma>1$, system (3) would have three equilibrium points, including $(0,0,0),(\gamma-1, \sqrt{\gamma-1}, \sqrt{\gamma-1})$, and $(\gamma-1,-\sqrt{\gamma-1},-\sqrt{\gamma-1})$.

(2) If $\gamma \leq 1$, system (3) would only have one equilibrium point, $(0,0,0)$.

The local stability of the equilibrium point is determined by the roots of the characteristic equation, and the equilibrium point is stable if the all the roots of the characteristic equation have negative real parts. The characteristic equation can be expressed as $\operatorname{det}(\lambda I-J)=0$, where $\lambda, I$, and $J$ represent the eigenvalue, identity matrix, and Jacobian matrix, 
respectively. In fact, the stability of the equilibrium point is usually judged using Routh-Hurwitz stability criterion [19] as solving the characteristic equation is very difficult for high dimensional dynamic system. (3) is

The Jacobian matrix at the equilibrium points of system

$$
J=\left[\begin{array}{ccc}
-1 & \widetilde{\omega}_{0} & \widetilde{i}_{q_{0}} \\
-\widetilde{\omega}_{0} & -1 & -\widetilde{i}_{d_{0}}+\gamma \\
0 & \sigma & -\sigma
\end{array}\right] .
$$

Substituting the Jacobian matrix and equilibrium point, $\left(\widetilde{\omega}_{0}^{2}, \widetilde{\omega}_{0}, \widetilde{\omega}_{0}\right)$, into the characteristic equation, $\operatorname{det}(\lambda I-J)=0$, we obtain

$$
\begin{aligned}
\lambda^{3}+ & (\sigma+2) \lambda^{2}+\left(1+\sigma \widetilde{\omega}_{0}^{2}-\sigma \gamma+\widetilde{\omega}_{0}^{2}+2 \sigma\right) \lambda+3 \sigma \widetilde{\omega}_{0}^{2} \\
& -\sigma \gamma+\sigma=0
\end{aligned}
$$

According to Routh-Hurwitz stability criterion, the sufficient condition of the local stability of the equilibrium points is

$$
\begin{aligned}
& \sigma>-2, \\
& 3 \sigma \widetilde{\omega}_{0}^{2}-\gamma \sigma+\sigma>0, \\
& (\sigma+2)\left(1+\sigma \widetilde{\omega}_{0}^{2}-\sigma \gamma+\widetilde{\omega}_{0}^{2}+2 \sigma\right)-\left(3 \widetilde{\omega}_{0}^{2}+\gamma-1\right) \sigma \\
& \quad>0 .
\end{aligned}
$$

By the bifurcation theory of the equilibrium points, the equilibrium points may lose stability when the parameters pass through the key values and the bifurcation behavior occurs [20-23]. In order to obtain the conditions of Hopf bifurcation, setting $\lambda=n j(n \neq 0)$ and substituting it into characteristic equation (6), we obtain

$$
\begin{aligned}
& -n^{3} j-(\sigma+2) n^{2}+\left(1+\sigma \widetilde{\omega}_{0}^{2}-\sigma \gamma+\widetilde{\omega}_{0}^{2}+2 \sigma\right) n j \\
& +3 \sigma \widetilde{\omega}_{0}^{2}-\sigma \gamma+\sigma=0,
\end{aligned}
$$

equating real and imaginary parts of the equation

$$
\begin{aligned}
& n^{2}=1+\sigma \widetilde{\omega}_{0}^{2}-\sigma \gamma+\widetilde{\omega}_{0}^{2}+2 \sigma, \\
& n^{2}=\frac{\left(3 \sigma \widetilde{\omega}_{0}^{2}-\sigma \gamma+\sigma\right)}{(\sigma+2)} .
\end{aligned}
$$

Then, if Hopf bifurcation occurs at the equilibrium point of (3), the following conditions must be satisfied:

$$
\begin{aligned}
\sigma+2 & >0, \\
1+\sigma \widetilde{\omega}_{0}^{2}-\sigma \gamma+\widetilde{\omega}_{0}^{2}+2 \sigma & >0, \\
\left(-\sigma^{2}-2\right) \omega_{0}^{2}+(\gamma-2) \sigma^{2}+(\gamma-4) \sigma-2 & =0 .
\end{aligned}
$$

In addition, if $\gamma>1$, system (3) would have two stable equilibrium points and one unstable equilibrium point and if $\gamma<1$, system (3) would only have one stable equilibrium point, and the pitchfork bifurcation occurs at $\gamma=1$.

(B) When considering the external load, namely, $L_{d}=L_{q}$, $\tilde{u}_{d}=0, \widetilde{u}_{q}=0$, and $\widetilde{T}_{L} \neq 0$, this is a special case that the control inputs of the system are removed after the motor runs for a period of operation. Let $u=\widetilde{T}_{L}$, and then system (2) becomes

$$
\begin{aligned}
& \frac{\mathrm{d} \tilde{i}_{d}}{\mathrm{~d} \tilde{t}}=-\widetilde{i}_{d}+\widetilde{\omega}_{q}, \\
& \frac{\mathrm{d} \widetilde{i}_{q}}{\mathrm{~d} \widetilde{t}}=-\widetilde{i}_{q}-\widetilde{\omega}_{d}+\gamma \widetilde{\omega}, \\
& \frac{\mathrm{d} \widetilde{\omega}}{\mathrm{d} \widetilde{t}}=\sigma\left(\widetilde{i}_{q}-\widetilde{\omega}\right)-u .
\end{aligned}
$$

By analyzing the equilibrium points, $\left(\widetilde{i}_{d_{0}}, \widetilde{i}_{q_{0}}, \widetilde{\omega}_{0}\right)$, of system (11), we obtain

$$
\left\{\begin{array} { l } 
{ - \widetilde { i } _ { d _ { 0 } } + \widetilde { \omega } _ { 0 } \tilde { i } _ { q _ { 0 } } = 0 } \\
{ - \widetilde { i } _ { q _ { 0 } } - \widetilde { \omega } _ { 0 } \widetilde { i } _ { d _ { 0 } } + \gamma \widetilde { \omega } _ { 0 } = 0 } \\
{ \sigma ( \widetilde { i } _ { q _ { 0 } } - \widetilde { \omega } _ { 0 } ) = 0 }
\end{array} \Longrightarrow \left\{\begin{array}{l}
\widetilde{i}_{d_{0}}=\widetilde{\omega}_{0}^{2}+\frac{u \widetilde{\omega}_{0}}{\sigma} \\
\widetilde{i}_{q_{0}}=\widetilde{\omega}_{0}+\frac{u}{\sigma} \\
\widetilde{\omega}_{0}^{3}+\frac{u}{\sigma} \omega_{0}^{2}+(1-\gamma) \widetilde{\omega}_{0}-\frac{u}{\sigma}=0 .
\end{array}\right.\right.
$$

According to the relationship between $\widetilde{i}_{d_{0}}, \widetilde{i}_{q_{0}}$, and $\widetilde{\omega}_{0}$, the equilibrium point of (11) can be written as $\left(\widetilde{\omega}_{0}^{2}+u \widetilde{\omega}_{0} / \sigma, \widetilde{\omega}_{0}+\right.$ $\left.u / \sigma, \widetilde{\omega}_{0}\right)$.

The Jacobian matrix at equilibrium point is

$$
J=\left[\begin{array}{ccc}
-1 & \widetilde{\omega}_{0} & \tilde{i}_{q_{0}} \\
-\widetilde{\omega}_{0} & -1 & -\tilde{i}_{d_{0}}+\gamma \\
0 & \sigma & -\sigma
\end{array}\right] .
$$

Substituting the Jacobian matrix and equilibrium into characteristic equation, $\operatorname{det}(\lambda I-J)=0$, we obtain

$$
\lambda^{3}+a_{1} \lambda^{2}+a_{2} \lambda+a_{3}=0
$$

where

$$
a_{1}=\sigma+2
$$




$$
\begin{aligned}
& a_{2}=(1+\sigma) \widetilde{\omega}_{0}^{2}+u \widetilde{\omega}_{0}+(2-\gamma) \sigma+1 \\
& a_{3}=3 \sigma \widetilde{\omega}_{0}^{2}+u \widetilde{\omega}_{0}+(u-r+1) \sigma .
\end{aligned}
$$

According to the Routh-Hurwitz criteria, the local stability condition of the equilibrium can be written as

$$
\begin{array}{r}
\sigma+2>0 \\
3 \sigma \widetilde{\omega}_{0}^{2}+u \widetilde{\omega}_{0}+(u-\gamma+1) \sigma>0 \\
\sigma\left(2+2 \sigma+(\sigma+2) \widetilde{\omega}_{0}^{2}+u \widetilde{\omega}_{0}-\gamma(\sigma+1)\right)+2>0 .
\end{array}
$$

If Fold bifurcation occurs at the equilibrium point of (11), the following conditions must be satisfied:

$$
\begin{aligned}
& \widetilde{\omega}_{0}^{3}+\frac{u}{\sigma} \omega_{0}^{2}+(1-\gamma) \widetilde{\omega}_{0}+\frac{u}{\sigma}=0 \\
& 3 \sigma \widetilde{\omega}_{0}^{2}+u \widetilde{\omega}_{0}+(u-\gamma+1) \sigma=0 .
\end{aligned}
$$

If Hopf bifurcation occurs at the equilibrium point of (11), the following conditions must be satisfied:

$$
\begin{array}{r}
\sigma+2>0 \\
(1+\sigma) \widetilde{\omega}_{0}^{2}+u \widetilde{\omega}_{0}+(2-\gamma) \sigma+1>0 \\
\widetilde{\omega}_{0}^{3}+\frac{u}{\sigma} \omega_{0}^{2}+(1-\gamma) \widetilde{\omega}_{0}+\frac{u}{\sigma}=0 \\
\sigma\left(2+2 \sigma+(\sigma+2) \widetilde{\omega}_{0}^{2}+u \widetilde{\omega}_{0}-\gamma(\sigma+1)\right)+2=0 .
\end{array}
$$

\section{Simulation Results}

This section presents some numerical simulation results to verify the existences of bifurcation behaviors which have been derived in the above section and the complicated dynamic behaviors of the PMSM with/without external load. For the sake of simplicity, let $x=\widetilde{i}_{d}, y=\widetilde{i}_{q}$, and $z=\widetilde{\omega}$.

(A) In this part, the inherent characteristics, namely, $\widetilde{u}_{d}=$ 0 , $\widetilde{u}_{q}=0$, and $\widetilde{T}_{L}=0$, of the PMSM will be discussed by numerical simulation. The double-parameter bifurcation curves (see Figure 1) of system (3) in $\gamma$ - $\sigma$ plane are obtained by continuation method, where "BP" and "subH" represent pitchfork bifurcation line and subcritical Hopf bifurcation line, respectively. The pitchfork bifurcation line together with subcritical Hopf bifurcation line divides the equilibrium parameter plane into three regions, namely, "A"; "B"; and "C," where the equilibrium has different characteristics in different regions. There are only one stable equilibrium point in " $\mathrm{A}$ " and two stable equilibrium points and one unstable equilibrium point in " $\mathrm{B}$ " and three unstable equilibrium points in "C."

By fixing the parameter $\sigma=10$ and letting parameter $\gamma$ cross through the three regions (namely, $\gamma$ is the bifurcation parameter which ranges from 0 to 20), the equilibrium line is presented in Figure 2, where the solid and the dotted curves represent the stable and unstable equilibrium points, respectively. " $E_{1}$ ”; “ $E_{2}$ ”; “ $E_{3}$ ”; “ $E_{1}^{\prime}$ ” represent equilibrium

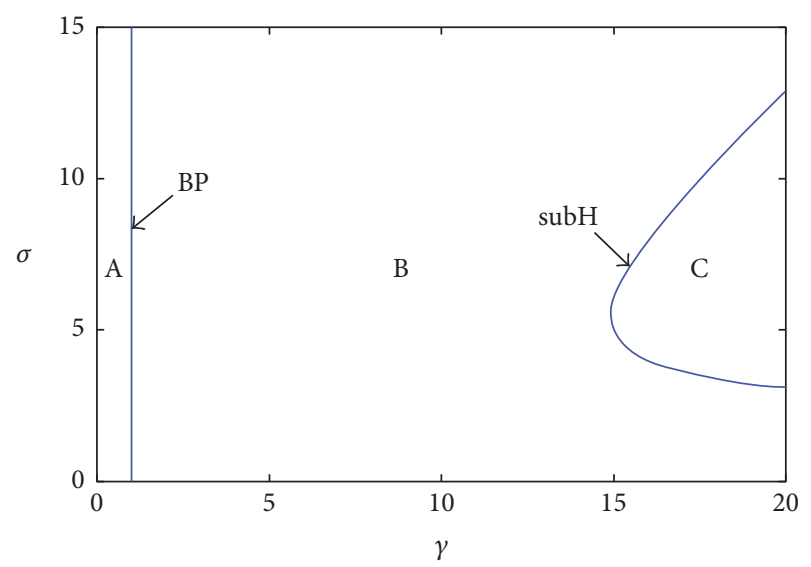

FIgURE 1: Bifurcation set in $\gamma-\sigma$ plane.

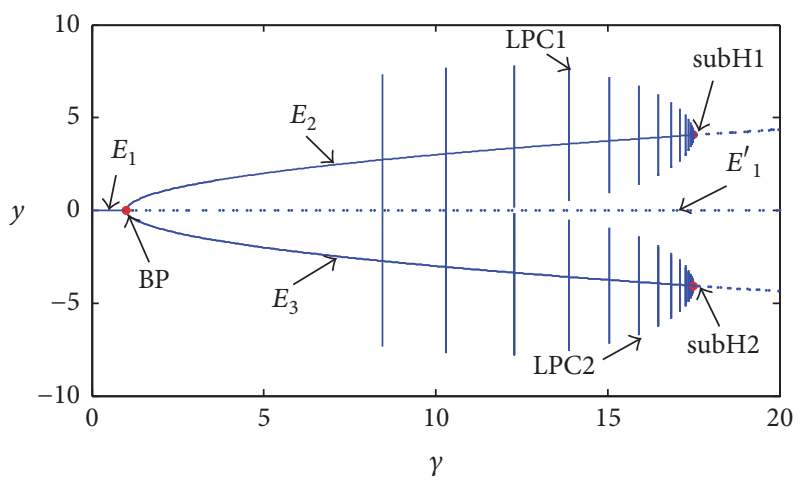

FIgURE 2: Equilibrium line for $\sigma=10$.

points, and "BP" $(\gamma=1)$ represents pitchfork bifurcation point, "subH1" and "subH2" $(\gamma=17.59)$ represent subcritical Hopf bifurcation and "LPC1" and "LPC2" represent the corresponding unstable limit cycle, respectively. From Figure 2, we obtain the following:

(a) If $0<\gamma<1$, there is only one stable equilibrium point for each $\gamma$; see Figure 3 .

(b) If $1<\gamma<17.59$, there are one stable and two unstable equilibrium points for each $\gamma$; see Figure 4 .

(c) If $17.59<\gamma<20$, there are three unstable equilibrium points for each $\gamma$.

Fix the parameter $\sigma=10$ and let parameter $\gamma$ be the bifurcation parameter which ranges from 0 to 20 . Figure 5 shows the bifurcation diagram which describes the global dynamic behaviors of the PMSM and the ordinate and the abscissa represent the motor angular speed and parameter $\gamma$ respectively. From Figure 5, we obtain the following.

(a) If $0<\gamma<1$, the trajectory eventually stabilized at " $E_{1}$ ".

(b) If $1<\gamma<2.1$, the trajectory eventually stabilized at " $E_{3}$ ".

(c) If $2.1<\gamma<5.4$, the trajectory eventually stabilized at " $E_{2}$ ". 


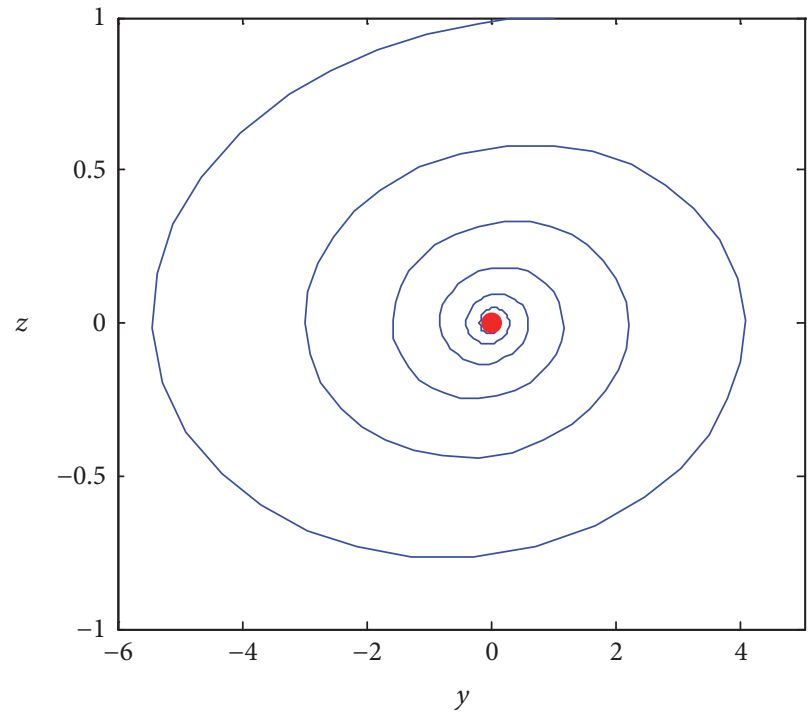

(a)

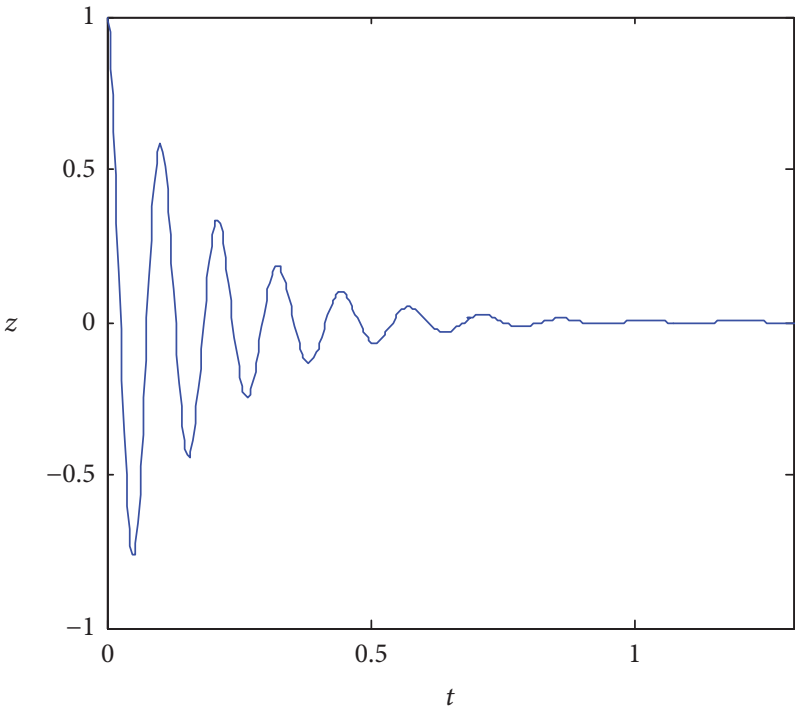

(b)

Figure 3: Stable equilibrium point for $\gamma=0.5$. (a) Phase diagram. (b) Time history.

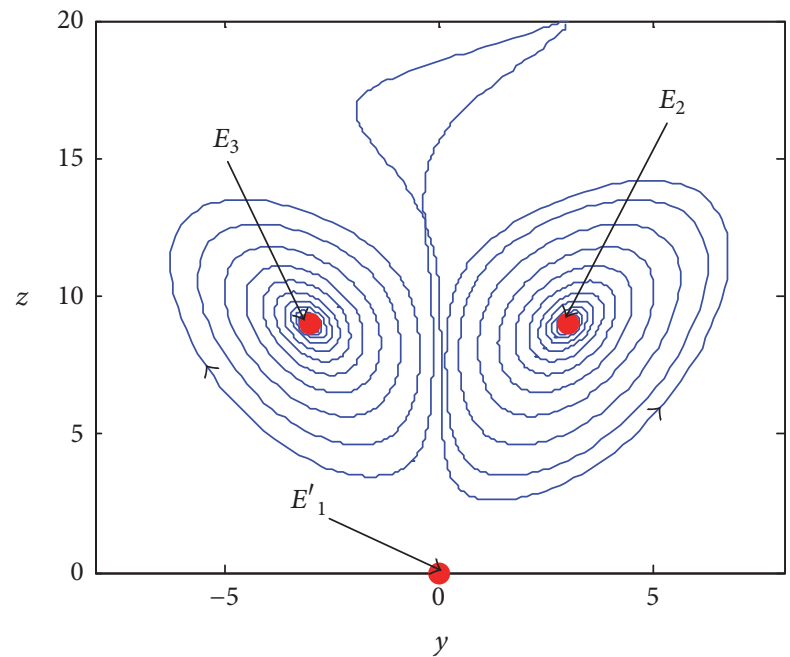

FIgURE 4: Multiequilibrium coexistence phenomenon for $\gamma=10$.

(d) If $5.4<\gamma<15$, the trajectory eventually stabilized at " $E_{3}$ ".

(e) If $15<\gamma<20$, the trajectory is attracted by the chaotic attractor.

It should be noted that when parameter $(\gamma)$ is selected between 13 and 15, the trajectory of the PMSM system is still asymptotically stable at equilibrium point " $E_{3}$ ", but the convergence speed is markedly slower than parameter $(\gamma)$ selected between 5.4 and 13. Before the trajectory is projected on the $Z-\gamma$ plane, 1000 points are deleted for each value of parameter $(\gamma)$ to eliminate the effect of initial condition and get steady-state numerical solution. As the PMSM system has

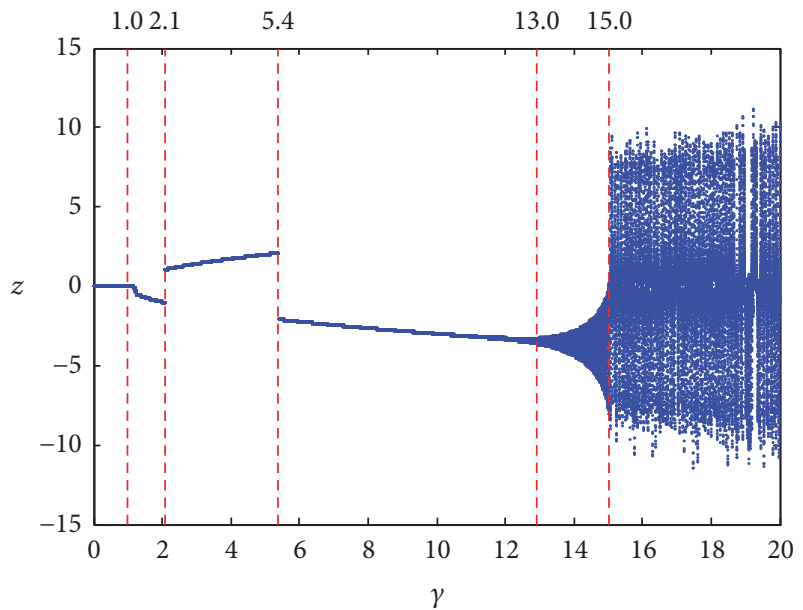

FIGURE 5: Bifurcation diagram of $z$ versus $\gamma$.

different convergence speed with the change of parameter $(\gamma)$, the effect of the initial condition is not completely eliminated between 13 and 15 even through 1000 points are deleted. In order to explain this phenomenon, parameter $(\gamma)$ is selected as 14 and the phase diagram and time history are obtained; see Figure 6. As can be seen, the trajectory is stable at the equilibrium point finally, but there exists long time transient process. If more points are deleted before projecting the trajectory on the $Z-\gamma$ plane, the transient process can be eliminated.

In order to prove the chaotic motion, parameter $(\gamma)$ is selected as 18 which falls into the chaotic region $(15<\gamma<$ 20 ) and three Lyapunov exponents of the PMSM system are calculated; see Figure 7(a). As can be seen, the maximum Lyapunov exponent, labelled as "Le $e_{1}$," is equal to 0.414 and the 


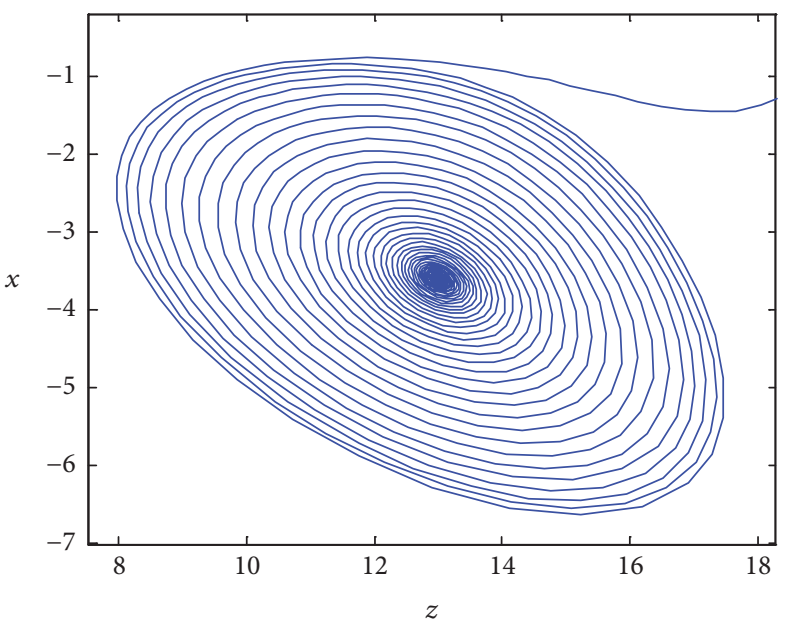

(a)

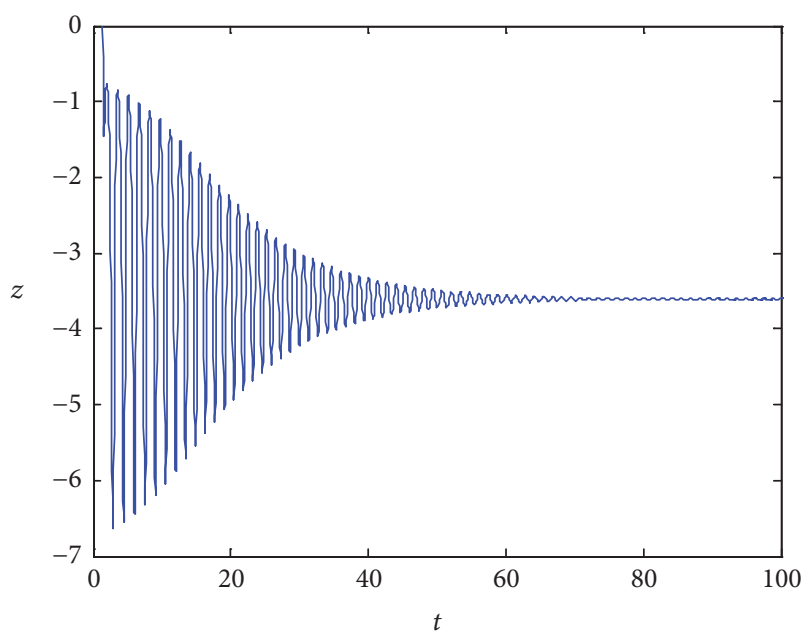

(b)

Figure 6: Phase diagram and time history for $\gamma=14$. (a) Phase diagram. (b) Time history.

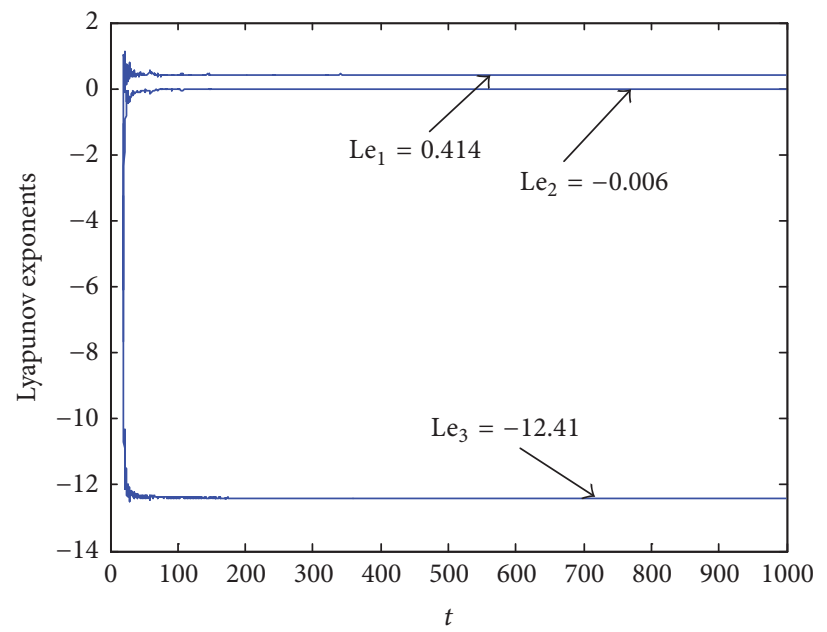

(a)

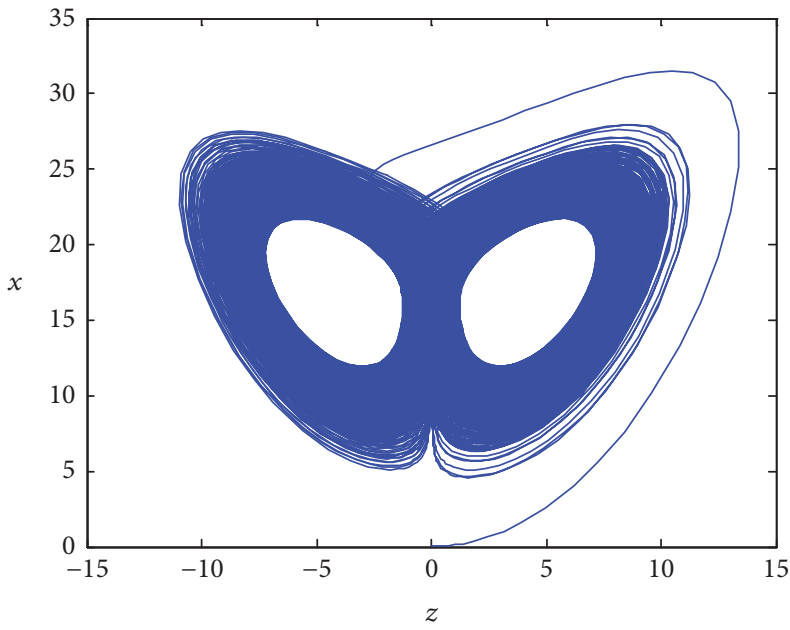

(b)

FIgURE 7: Lyapunov exponents and phase diagram for $\gamma=18$. (a) Lyapunov exponents. (b) Phase diagram.

other two Lyapunov exponents are -0.06 and -12.41 , respectively. Meanwhile, the trajectory of attractor (see Figure 7(b)) likes "Butterfly" in the phase diagram. So the attractor when $\gamma$ is equal to 18 is chaotic motion and can be named as doublescroll chaotic attractor.

It is easy to identify that the PMSM exhibits various dynamic behaviors in different parameter regions and the unstable dynamic behavior may occur when the parameter passes through key values. If there is only one stable equilibrium in the parameters regions, the PMSM can work stably, and if the stable equilibrium point and unstable equilibrium point are coexistent in the parameters regions, the PMSM trajectory may be attracted by one of the stable equilibrium points; meanwhile, chaotic motion may happen, and if there is no stable equilibrium points in the parameters regions, the PMSM cannot work stably.
To verify the correctness of the above research, Table 1 lists a set of parameters of PMSM [24], and the multiequilibrium coexistence phenomenon (see Figure 8 ) is obtained by simulation as the parameters of the PMSM fall in region "B."

(B) In this part, the dynamic behaviors of the PMSM with external load, namely, $\tilde{u}_{d}=0, \widetilde{u}_{q}=0$, and $\widetilde{T}_{L}=u \neq 0$, will be discussed by the bifurcation set and bifurcation line as the PMSM may not be able to work stably when unstable equilibrium point appears in the corresponding parameters regions and the stability of the equilibrium point can be judged by the bifurcation set and bifurcation line, which is discussed in (A).

The bifurcation set of the equilibrium point of (11) (see Figure 9) describes the bifurcation and stability characteristics of the equilibrium point in $u-\sigma$ plane, where the parameter $\gamma$ is fixed as 4.738 and the PMSM itself has one 
TABLE 1: Parameter values of the PMSM system model [24].

\begin{tabular}{lccccc}
\hline Parameter & Value & Unit & Parameter & Value & Unit \\
\hline$\frac{L_{d}}{L_{q}}$ & 1.01 & $\mathrm{mH}$ & $J$ & $4.8 \times 10^{-5}$ & \\
$\psi_{R}$ & & & $p_{n}$ & 4 & \\
$R_{1}$ & 0.06784 & $\mathrm{Nm} / \mathrm{A}$ & $\Omega$ & 0.01619 & $\mathrm{~N} / \mathrm{rad} / \mathrm{s}$ \\
\hline
\end{tabular}

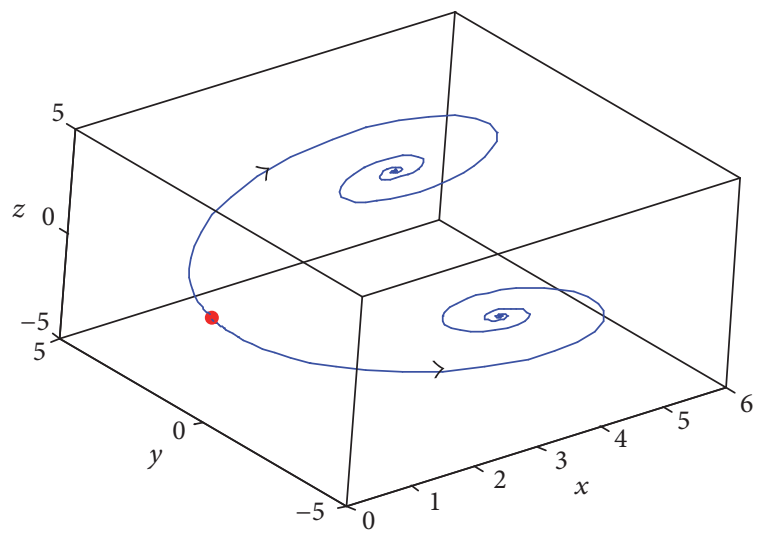

FIGURE 8: Multiequilibrium coexistence phenomenon for $\gamma=4.378$ and $\sigma=1.419$.

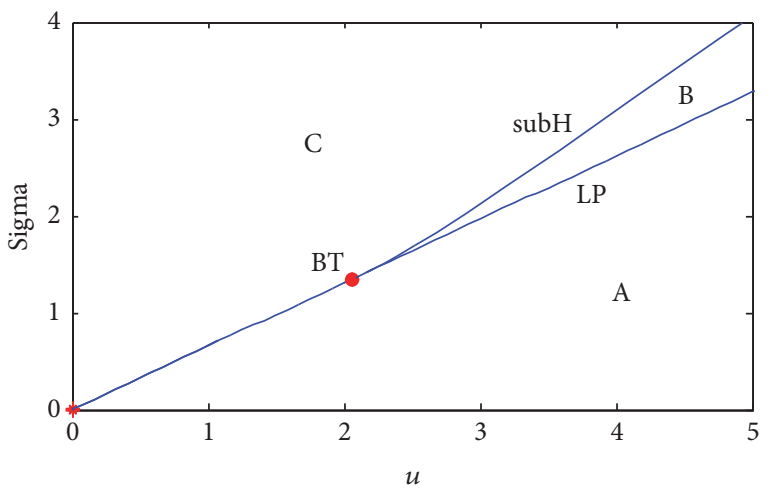

FIGURE 9: Bifurcation set in $u-\sigma$ plane.

unstable equilibrium point as Figure 1 shows. "BT" represents the Bogdanov-Takens bifurcation of the equilibrium. The fold bifurcation line, labelled as "LP," together with subcritical Hopf bifurcation line, labelled as "subH," divided the equilibrium parameter plane into three regions, namely, " $\mathrm{A}$ " to "C." There is only one stable equilibrium point in "A," and the characteristics of this stable equilibrium point are not changed and two unstable equilibrium points appear when the parameters passes fold bifurcation line from " $A$ " to "B." There are one stable and two unstable equilibrium points in "B." When the parameters pass through subcritical Hopf bifurcation line from " $B$ " to "C," one of the unstable equilibrium points becomes stable; namely, there are two stable equilibriums and one unstable equilibrium in " $\mathrm{C}$."

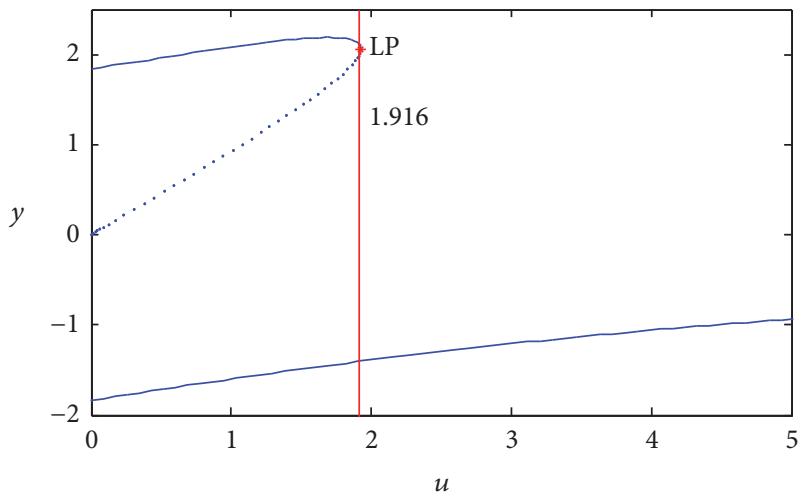

FIGURE 10: Bifurcation line in $u-y$ plane.

Obviously, the PMSM can work stably in "A" and may lose its stability in other regions.

For the given parameters in Table 1, the bifurcation line of PMSM with external load is drawn in Figure 10, which shows that the PMSM has three equilibrium points, two stable and one unstable, in $0<u<1.916$ and only one stable equilibrium point in $u>1.916$. When the load $u$ passes the fold bifurcation point, the "Jump" behavior, which is unstable, may happen. The PMSM can still work stably when $u>1.916$ even though it has unstable inherent parameters.

\section{Conclusion}

The complicated dynamic behaviors of the PMSM with/ without external load are studied. The stability conditions and bifurcation conditions of equilibrium points have been derived by using Routh-Hurwitz stability criterion and bifurcation theory, respectively. The characteristics of equilibrium points are analyzed by bifurcation set, and pitchfork bifurcation, fold bifurcation, and subcritical Hopf bifurcation lines are obtained by numerical simulation. The global dynamic behaviors of the PMSM are studied by using bifurcation diagram. It is shown that if there is only one stable equilibrium in the parameters regions, the PMSM can work stably, and if multiequilibrium coexistence phenomenon appears in the parameters regions, the trajectory may be attracted by one of the stable equilibriums; meanwhile, the PMSM may not be able to work stably as chaotic motion happens in this region, and if there is no stable equilibrium in the parameters regions, the PMSM can not work stably. It is also shown that the PMSM can still work stably in some load regions even though 
it has unstable parameters. To ensure the PMSMs work stably, the inherent parameters should be designed in the region which has only one stable equilibrium.

\section{Disclosure}

The results and opinions expressed in this article are those of the authors only and they do not necessarily represent those of the sponsors.

\section{Conflicts of Interest}

The authors declare that there are no conflicts of interest regarding the publication of this paper.

\section{Acknowledgments}

The authors disclosed receipt of the following financial support for the research, authorship, and/or publication of this article: financial support to complete this study was provided in part by the National Natural Science Foundation of China under no. 51275212, by the Natural Science Foundation of the Jiangsu Higher Education Institutions of China under no. 13KJA580001, and by Research Innovation Program for College Graduates of Jiangsu Province of China under no. KYZZ16_0332.

\section{References}

[1] A. Draou, "A simplified sliding mode controlled electronic differential for an electric vehicle with two independent wheel drives," Energy \& Power Engineering, vol. 5, no. 6, pp. 416-421, 2013.

[2] H. Yu, J. Xi, F. Zhang, and Y. Hu, "Research on gear shifting process without disengaging clutch for a parallel hybrid electric vehicle equipped with AMT," Mathematical Problems in Engineering, vol. 2014, Article ID 985652, 12 pages, 2014.

[3] Z. Lu, X. Sun, and J. Zhang, "Design and control of disc PMSM directly driven wheel for tramcar," Advances in Mechanical Engineering, vol. 2014, Article ID 747636, 8 pages, 2014.

[4] A. Kreim and U. Schäfer, "An approach to an optimal design of permanent magnet synchronous machines for battery electric vehicles," in Proceedings of 27th World Electric Vehicle Symposium and Exhibition, EVS 2014, esp, November 2013.

[5] G. Bramerdorfer, W. Amrhein, S. M. Winkler, and M. Affenzeller, "Identification of a nonlinear PMSM model using symbolic regression and its application to current optimization scenarios," pp. 628-633.

[6] W. Chao, G. Qiang, H. Yuanlong, H. Runmin, and M. Hao, "Adaptive complementary fuzzy self-recurrent wavelet neural network controller for the electric load simulator system," Advances in Mechanical Engineering, vol. 8, no. 3, pp. 1-12, 2016.

[7] P. Song, CL. Xia, Y. Yan et al., "Matrix converter fed permanent magnet synchronous motor drive system," Proceedings of the CSEE, vol. 31, no. 9, pp. 58-65.

[8] H. S. Li, Y. Zhou, Q. Cao et al., Mathematical deduction and stability analysis of PMSm open-loop control system, pp. 36-43, 2014.

[9] M. Hinkkanen, T. Tuovinen, L. Harnefors, and J. Luomi, "A combined position and stator-resistance observer for salient
PMSM drives: Design and stability analysis," IEEE Transactions on Power Electronics, vol. 27, no. 2, pp. 601-609, 2012.

[10] R. Ortega, L. Praly, A. Astolfi, J. Lee, and K. Nam, "Estimation of rotor position and speed of permanent magnet synchronous motors with guaranteed stability," IEEE Transactions on Control Systems Technology, vol. 19, no. 3, pp. 601-614, 2011.

[11] M. Novák, A. Flah, J. Novák et al., "Online high-speed pmsm parameters estimation and stability analysis," Acta Polytechnica Hungarica, vol. 11, no. 9, pp. 75-94, 2014.

[12] A. K. Yadav, P. Gaur, and P. Saxena, "Robust stability analysis of pmsm with parametric uncertainty using kharitonov theorem," Journal of Electrical Systems, vol. 12, no. 2, pp. 258-277, 2016.

[13] X. Guo, X. Wen, F. Zhao, X. Song, and X. Zhuang, "PI parameter design of the flux weakening control for PMSM based on small signal and transfer function," in Proceedings of 12th International Conference on Electrical Machines and Systems, ICEMS 2009, jpn, November 2009.

[14] S. C. Chang and B. C. Lin, "Dither signal effects on quenching chaos of a permanent magnet synchronous motor in electric vehicles," Journal of Vibration \& Control, vol. 17, no. 12, pp. 19121918, 2011.

[15] S.-C. Chang and H.-P. Lin, "Study on controlling chaos of permanent magnet synchronous motor in electric vehicles," International Journal of Vehicle Design, vol. 58, no. 2-4, pp. 387398, 2012.

[16] X. Wang and C. S. Suh, "Nonlinear time-frequency control of PM synchronous motor instability applicable to electric vehicle application," International Journal of Dynamics \& Control, vol. 4, no. 1, pp. 400-412, 2016.

[17] L. Zhong, J. B. Park, Y. H. Joo, and G.-R. Zhang Bo Chen, "Bifurcations and chaos in a permanent magnet synchronous motor," IEEE Transactions on Circuits \& Systems I Fundamental Theory \& Applications, vol. 49, no. 3, pp. 383-387, 2002.

[18] Z. J. Jing, Y. Chang, and G. R. Chen, "Complex dynamics in a permanent-magnet synchronous motor model," Chaos, Solitons \& Fractals, vol. 22, no. 4, pp. 831-848, 2004.

[19] J. A. Pereda, L. A. Vielva, A. Vegas et al., "Analyzing the stability of the FDTD technique by combining the von Neumann method with the Routh-Hurwitz criterion," IEEE Transactions on Microwave Theory \& Techniques, vol. 49, no. 2, pp. 377-381, 2001.

[20] F. Gordillo, F. Salas, R. Ortega, and J. Aracil, "Hopf bifurcation in indirect field-oriented control of induction motors," Automatica. A Journal of IFAC, the International Federation of Automatic Control, vol. 38, no. 5, pp. 829-835, 2002.

[21] K. T. Alligood, T. D. Sauer, and J. A. York, Chaos: An Introduction to Dynamical Systems, Springer, New York, NY, USA, 1996.

[22] P. Liu, J. Shi, and Y. Wang, "Imperfect transcritical and pitchfork bifurcations," Journal of Functional Analysis, vol. 251, no. 2, pp. 573-600, 2007.

[23] B. Fiedler, S. Yanchuk, V. Flunkert et al., "Delay stabilization of rotating waves near fold bifurcation and application to alloptical control of a semiconductor laser," Physical Review E. Statistical, Nonlinear, and Soft Matter Physics, vol. 77, no. 6, Article ID 066207, 066207, 9 pages, 2008.

[24] Z. Deng, J. Shang, and X. Nian, "Synchronization controller design of two coupling permanent magnet synchronous motors system with nonlinear constraints," ISA Transactions, vol. 59, pp. 243-255, 2015. 


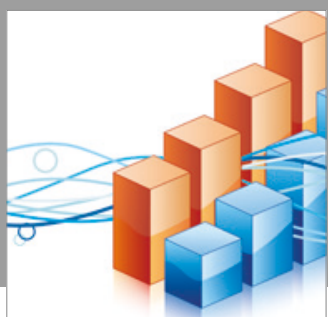

Advances in

Operations Research

vatersals

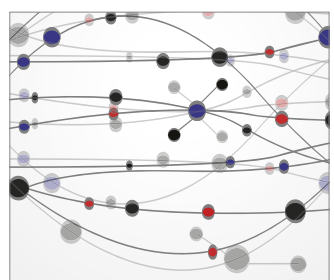

\section{The Scientific} World Journal
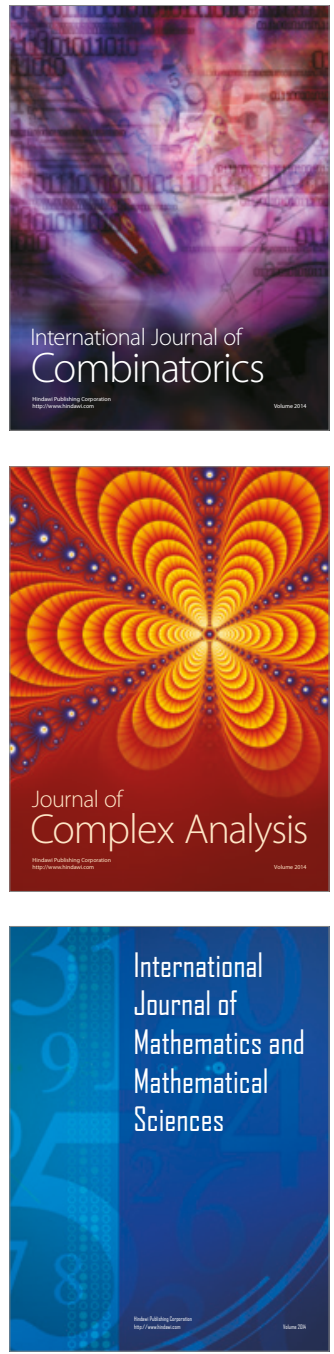
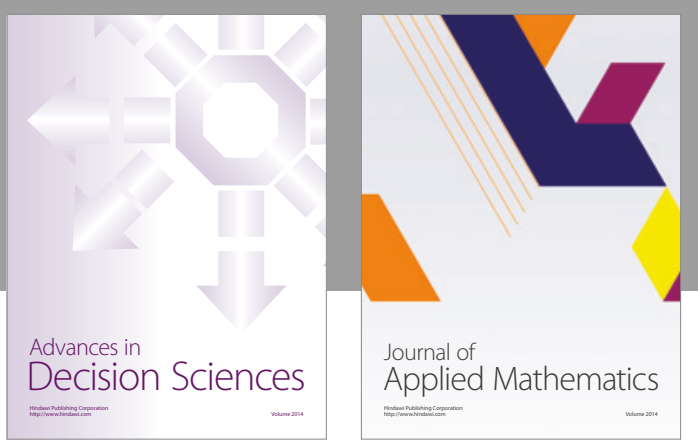

Algebra

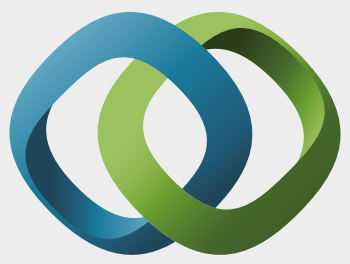

\section{Hindawi}

Submit your manuscripts at

https://www.hindawi.com
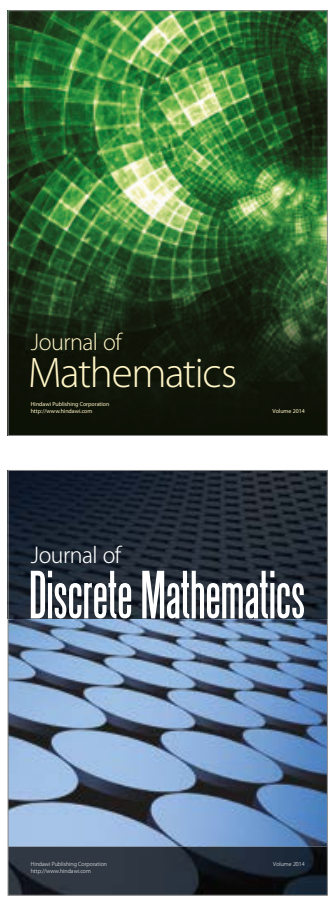

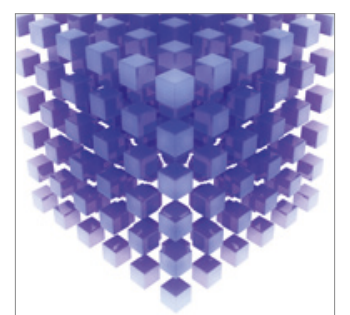

Mathematical Problems in Engineering
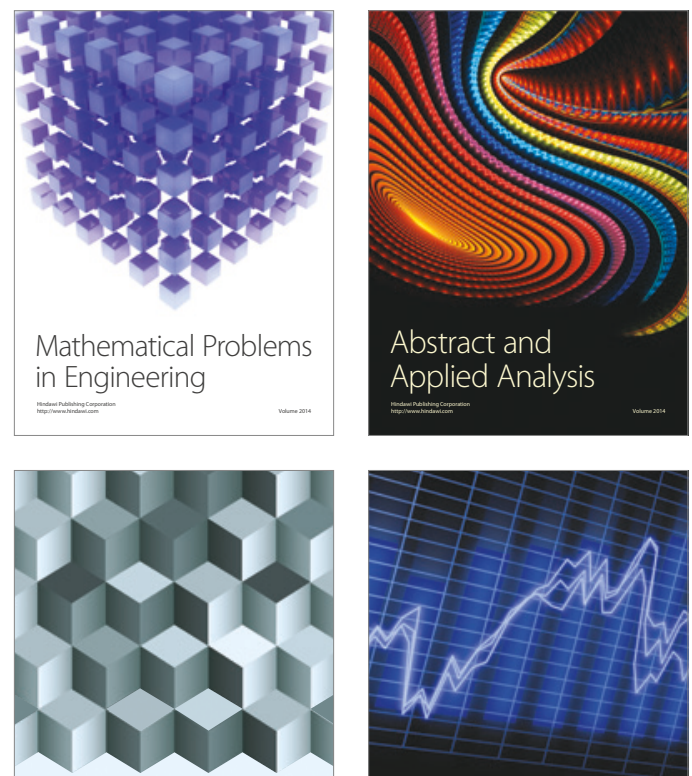

Journal of

Function Spaces

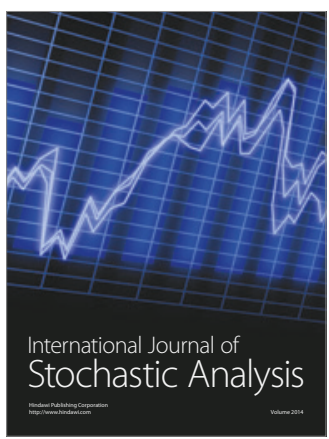

Probability and Statistics
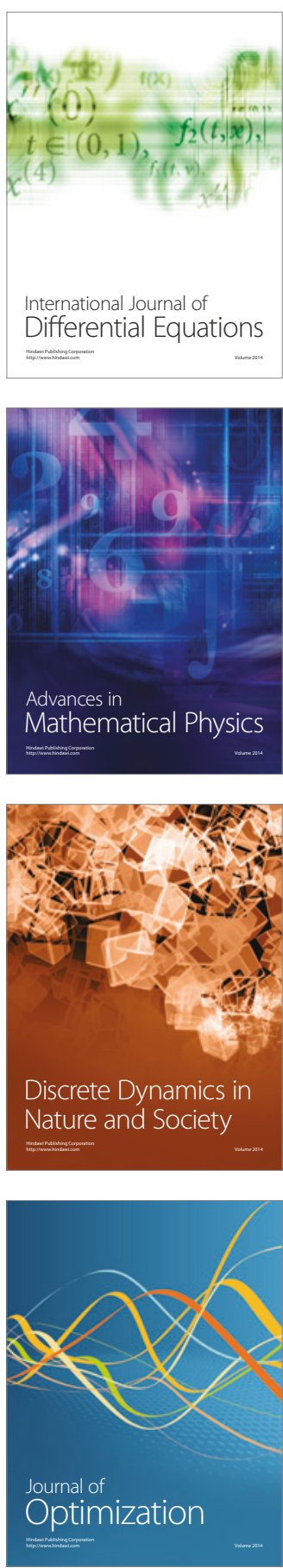